\title{
LA ATALANTA DE DOCTOR ZAY: TESTIMONIO DEL USO Y DE LA RECREACIÓN DE LA MITOLOGÍA CLÁSICA POR PARTE DE LAS ESCRITORAS NORTEAMERICANAS DEL SIGLO XIX
}

\author{
MARÍA DOLORES NARBONA CARRIÓN \\ Universidad de Málaga
}

\section{RESUMEN}

Este artículo destaca el mérito de una escritora victoriana norteamericana, Elizabeth Stuart Phelps (1844-1911), al demostrar tener suficientes conocimientos sobre mitología como para usarlos con maestría en sus escritos literarios en una época en que a las mujeres se les negaba el acceso a ciertos niveles de formación. Concretamente, analizo la nueva versión del mito de Atalanta que muestra en su novela Doctor Zay con el fin de hacer descubrir a las mujeres nuevas posibilidades para su futuro.

Palabras clave: Literatura norteamericana, mujeres escritoras, mitología, reforma social, mujeres doctoras.

\begin{abstract}
This article highlights the merit of a Victorian American writer, Elizabeth Stuart Phelps (1844-1911), as she had enough classical knowledge about mythology to use it skilfully in her literary writing at a time when women were not allowed access to a certain level of education. I concretely analyse the new version of the myth of Atalanta that she portrays in her novel Doctor Zay with the purpose of making women open their eyes to new possibilities for their lives.
\end{abstract}

Key words: American literature, women writers, mythology, social reform, women doctors.

En la literatura norteamericana hubo un período denominado "Victoriano" en que las escritoras gozaron de gran fama a pesar de los muchos obstáculos que encontraban en su camino. Así lo reconocen críticos como Susan Coultrap-McQuin o Lawrence Buell, quien destaca además el hecho de que fueron las mujeres antes que los hombres quienes se 
dedicaron profesionalmente a la labor de la escritura en Norteamérica (Buell, 1986: 195). No obstante, su estilo se ha venido estudiando de forma demasiado superficial, fijándose casi exclusivamente en sus rasgos románticos, dulces y sentimentales, que a su vez se suelen asociar, como señala Jane Tompkins, con trivialidades consideradas propias del mundo femenino (Tompkins, 1985: 123). Ciertamente, las características mencionadas estaban presentes en muchas obras literarias escritas por mujeres decimonónicas, pero un análisis detallado de las mismas que tenga en cuenta también el contexto en que se enmarcan y la vida de sus autoras nos revela que, detrás de su aspecto inocente, se escondían mensajes subversivos que así llegaban sin dificultad a sus lectoras. Podríamos decir que muchas de estas mujeres escribían de forma alegórica para transmitir un contenido que, de haber sido expuesto más claramente, habría topado con diferentes censuras: desde la de los propios hombres de la casa, que eran quienes por regla general proporcionaban el dinero a las mujeres para comprarlo todo - y, por ende, también los libros-, hasta la de las instituciones y la crítica, que no sólo podían acabar con las profesiones de estas escritoras, sino también con su reputación, por alejarse —e invitar a otras a alejarse — del ideal femenino de la época. Éste no era otro que el de la "True Woman”, también denominado “Ángel de la casa", cuyas virtudes principales giraban en torno a la pureza, la obediencia, la domesticidad y la sumisión, como concluye Barbara Welter en su amplio y conocido estudio sobre las mujeres norteamericanas del siglo XIX, Dimity Convictions; The American Woman in the Nineteenth Century (Welter, 1985: 21).

En este artículo nos vamos a fijar en una de las muchas escritoras que consiguieron una gran fama en sus días por sus dotes artísticas y que merecen ser re-evaluadas en el presente también por su valor al arriesgarse a cambiar su entorno por medio de su obra. Se trata de Elizabeth Stuart Phelps (1844-1911), autora que utilizó todos los géneros poesía, narrativa, teatro, relato y ensayo- para centrarse en lo que más le importaba: mejorar las condiciones de vida de las mujeres victorianas, estancadas en las imposiciones sociales de su época. A la valentía de este contenido hay que sumar la calidad de los escritos de Phelps, que han seguido atrayendo la atención de ciertos académicos, llegando al punto de que autoras como Lori Duin Kelly la consideren la versión femenina de Walt Whitman, que invita al sector femenino a cantar con ella "Song of Myself" (Duin-Kelly, 1983: 120). Como destaca la obra de Kathryn Gleadle, The Early Feminists. Radical Unitarians and the Emergence of the Women's Rights Movement, 1831-51, los unitarios y los transcendentalistas guardaban ciertas similitudes, fomentadas por el contacto existente entre ellos. En lo que concierne a la literatura, hay que destacar que ambos la consideraban como un destacado vehículo de denuncia para avanzar hacia la consecución de las reformas sociales necesarias en su entorno (Gleadle, 1995: 18, 55). Este mismo interés está claramente presente en los fundamentos de los escritos de Phelps — gran admiradora de transcendentalistas como Margaret Fuller-, que además afirma abiertamente: "The province of the artist is to portray life as it is; and life is moral responsibility . . . An artist can no more fling off the moral sense from this work than he can oust it from his private life" (Phelps, 1980: 263). Lori Duin Kelly destaca en su obra el hecho de que la crítica se haya fijado de manera superficial en sus escritos religiosos, olvidando su continuo esfuerzo por acabar con los estereotipos femeninos que impedían a las mujeres conseguir sus propias metas más allá de los mismos (Kelly, 1983: vii). 
Si es encomiable la labor de todo artista que consigue destacar por sus creaciones, el caso de Phelps, como el de otras escritoras afamadas contemporáneas suyas, lo es aún más si tenemos en cuenta lo difícil que era para ellas el acceso a la educación académica. Ya señalamos antes que en la época de la autora, las mujeres vivían a la sombra del ideal marcado por la "True Woman", que hacía que se les definiera en función de la figura masculina como hijas, esposas y madres. Para justificar tal delimitación, se basaban en datos "científicos" como su idoneidad para la procreación. Una de las consecuencias que ello traía consigo era que su formación se limitara al ámbito doméstico, sin que se les permitiera asistir a instituciones de enseñanza al mismo nivel que las de los hombres. Las afortunadas que conseguían algún tutor o maestro que las instruyera en casa tampoco estudiaban lo mismo que los hombres, lo que afectaba especialmente a asignaturas como el griego y las matemáticas, que consideraban que forzaban demasiado el delicado e inferior cerebro femenino.

No es de extrañar, pues, que coincida el que casi todas las escritoras decimonónicas que consiguieron crear obras de considerable calidad tuvieran al menos acceso a una biblioteca familiar donde pasaban horas leyendo, entre otras cosas, obras clásicas. Este fue el caso de Phelps, que creció en una familia erudita y tradicional que contaba con varios escritores: sus dos abuelos —Moses Stuart (1870-1852) y Eliakim Phelps (1790-1880)—, su madre Elizabeth (Stuart) Phelps (1815-1852), que escribía con el seudónimo H. Trusta-, y su padre -Austin Phelps (1820-1890). Pero tampoco ellos consideraron que Elizabeth tenía el mismo derecho que cualquier chico a asistir al seminario local de Andover, de forma que fue su padre quien se encargó de orientar su formación intelectual (Kessler, 1982: ii). ${ }^{1}$ Además, como muchas de las chicas de Andover, asistió a la Academia Abbot, y a la "School for Young Ladies" de la conservadora Mrs. Edwards. A pesar de todos estos sustitutos de una formación académica como la masculina, Phelps siguió sin tener acceso "oficial" a las materias antes mencionadas, de forma que su curriculum seguía carente de estudios clásicos porque estos, junto con la trigonometría, en palabras suyas, "se consideraban en aquellos días fuera del ámbito del intelecto femenino" ("[they were] thought, in those days, to be beyond the scope of the feminine intellect") (Phelps, 1980: 60-61).

No obstante, la rica obra literaria de Phelps demuestra que no se resignó a ser instruida como su entorno considerara más apropiado, sino que buscó la forma de familiarizarse tanto con los estudios clásicos, como con los autores que influirían en su forma de pensar, entre los que destacan Thomas De Quincey, William Wordsworth, Elizabeth Barrett Browning, George Eliot o John Stuart Mill. Como artista que era, Phelps supo dar forma a elementos tomados de la antigüedad clásica que mezclaba magistralmente con las ideas emanadas de sus reflexiones sobre estos grandes autores para "filtrárselas" a las mujeres con la intención de ayudarles a superarse y a salir de su estancamiento. De Stuart Mill, la

\footnotetext{
1 El hecho de que las jóvenes recibieran formación por parte de los padres era muy frecuente, especialmente en el seno de familias de alto nivel cultural. Entre los muchos ejemplos existentes, podemos mencionar el caso de Anne Bradstreet (1612-1672), que escribía además desde niña poemas para satisfacer a su padre y en su madurez contó con el apoyo de su esposo, Simon Bradstreet. Margaret Fuller también afirma haber recibido la mayor parte de formación cultural de manos de su padre. Así lo reconoce en Memoirs of Margaret Fuller Ossoli (1852) (Baym, 1999: 775-776).
} 
escritora recomienda abiertamente la lectura de The Subjection of Women (1869), según dejan ver las palabras que le dedica en su artículo "Unhappy Girls” (1871): “El sometimiento de las mujeres' [es] un libro que ninguna lectora debería estar tranquila hasta que lo hubiese visto" (Phelps, 1871: 1) (“'Subjection of Women' [is] a book which no reading woman in the land should rest till she has seen"). Pero, como decíamos al principio, Phelps también siguió la costumbre de transmitir sus ideas de una forma más velada en sus escritos de ficción, de forma que la propuesta de Stuart Mill $-\mathrm{y}$ de otras autoras como Mary Wollstonecraft, Elizabeth Barrett Browning o Margaret Fuller - sobre la necesidad de una relación igualitaria y de admiración mutua entre hombres y mujeres especialmente en el matrimonio (Stuart-Mill, 1997: 96) aparece de forma alegórica en varias de las obras de Phelps. En ocasiones, podemos decir que la práctica de Phelps se asemeja incluso al uso que Platón hace del mito "como instrumento para la difusión de las más profundas doctrinas filosóficas" (Bermejo, 1988: 9). De hecho, esta técnica no es exclusiva de la escritora, sino que, incluso en nuestros días, se reconoce cada vez más la necesidad de imágenes y mitos a través de los que ver reflejados los pensamientos, priorizando la imagen sobre la exposición directa de la idea. ${ }^{2}$

En este artículo nos vamos a centrar en Doctor Zay (1882), una de las obras más interesantes de Phelps, que no sólo expone sus propuestas de forma simbólica, sino que recurre además a un mito concreto: el de Atalanta. En ella, presenta a una de las protagonistas excepcionales que pueblan sus obras, en las que nos encontramos, por ejemplo, con mujeres que se dedican profesionalmente a la pintura - como ocurre en The Story of Avis (1877), donde también aparecen referencias mitológicas a Artemisa y a Ariadna-, y con otras que dejan su vida de opulencia para dedicarse a la reforma social en el mundo de la fábrica - es el caso de la protagonista de The Silent Partner (1871). El personaje principal de Doctor Zay, una mujer doctora que recorre a gran velocidad los bosques de su entorno para socorrer a sus enfermos y en cuyos esquemas mentales no tiene lugar el matrimonio, no podía encontrar mejor reflejo clásico que Atalanta, cuyo nombre le otorga además Phelps: la llama "Zaidee Atalanta Lloyd". Pero no sólo presenta estas "coincidencias", sino que tiñe su creación de referencias concretas al mito de Atalanta, que además subvierte en ciertos aspectos, como era propio de su estilo.

Para poder analizar los elementos del mito de Atalanta que aparecen en la mencionada novela, recordaremos primero en qué consiste. El mito fue recogido por las tradiciones griega y romana, y se aprecian ciertas incertidumbres y divergencias respecto a algunos detalles como su origen exacto (Gibson, 2003: 1), por lo que nos centraremos en los puntos comunes existentes. ${ }^{3}$ Este mito presenta a una mujer excepcional, una joven cuyo padre la abandona siendo bebé a las inclemencias de la naturaleza por no haber nacido varón. En este entorno resulta ser mejor tratada que en el anterior, ya que Atalanta es amamantada por una osa y va creciendo fuerte y con una hermosura fuera de serie. Dichos cuidados serían después proporcionados por cazadores, que respetan su promesa a Artemisa de permanecer

\footnotetext{
${ }^{2}$ Así lo reconoce Christine Dowing en su libro La diosa. Imágenes mitológicas de lo femenino. Véase, por ejemplo, la página 12 .

${ }^{3}$ Christine Downing señala que, cuando se indaga en el estudio de las diosas, es frecuente encontrar muchas variantes y ninguna versión fija, pues cada una de ellas ofrece una interpretación pertinente de la diosa y sus poderes (Downing, 1999: 38).
} 
por siempre virgen. Con ellos, Atalanta cazaba como uno más, llegando incluso a superarlos en una de sus gestas, la cacería del jabalí de Calidón. En este contexto masculino, Atalanta se acostumbró a defenderse de todo tipo de peligro, como cuando dos centauros intentaron violarla, situación que resolvió quitándoles la vida.

$\mathrm{Al}$ parecer sus proezas llegaron a oídos de su padre, que no debió echar en falta ya en ella las características tradicionalmente consideradas masculinas, según demostró sobradamente. Atalanta aceptó volver a su lado, pero se encontró con el problema de que su prometida virginidad estaba en peligro, ya que eran muchos los que, deslumbrados por su belleza, querían casarse con ella. La ágil cazadora pensó que un buen modo de mantener su consagración a Artemisa podría ser retar en una carrera a todo pretendiente, que debería ser castigado con la muerte si no le ganaba, o premiado con su mano, si llegaba a la meta antes que ella. Aunque podríamos pensar que nadie aceptaría tal competición, era tal el atractivo de la joven que fueron muchos los que perecieron por amor. Además, la astucia se fue agudizando hasta que entró en escena un joven también muy bello, Melanion, quien, más cauto que los anteriores, pidió colaboración a Afrodita, diosa del amor, para que le ayudara a vencer a su rival y a la vez amada. La diosa le proporcionó a Melanion tres manzanas de su jardín que éste arrojaba a Atalanta cada vez que le adelantaba. Ella se detenía a recogerlas - hay quien señala que no lo hacía tanto atraída por la manzana en sí como distraída por la belleza de Melanion- y era automáticamente adelantada por su contrincante. Así pues, Melanion, lejos de acabar en las fauces de la muerte, terminó en los brazos de Atalanta, que aceptó gustosamente casarse con alguien que realmente la amaba, admiraba y sentía como su igual. A partir de entonces se dedicarían los dos a lo mismo: a correr y a cazar por los bosques disfrutando de su mutua compañía y cariño.

El mito cuenta con segundas partes que narran cómo, quizá castigados por Afrodita, a quien Melanion olvidó dar las gracias por su ayuda, quizá simplemente porque profanaron el templo de Zeus, donde gozaron de su amor, los enamorados fueron transformados en leones. Pero esta parte no es relevante para el análisis que estamos haciendo de la obra de Phelps, ya que su contenido se detiene antes, en la victoria de Melanion, que es encarnado en Doctor Zay por Waldo Yorke.

Phelps debió considerar que el mito de Atalanta resultaba un trasfondo ideal para asociarlo con el modelo femenino que trataba de propagar con su novela Doctor Zay, ya que ella consideraba fundamental que las mujeres lucharan con fuerza por sacar adelante su auténtica vocación, haciendo oídos sordos a las imposiciones del patriarcado. Para la doctora Zay, como para sus coetáneas, ejercer la medicina resultaba muy complicado ya desde su propia formación, a la que se les solía denegar el acceso hasta entrado el final del siglo XIX —y aun entonces se encontraban con muchos obstáculos. ${ }^{4}$ Y no les era más fácil

\footnotetext{
${ }^{4}$ Como prueba de ello, existe la experiencia de Elizabeth Blackwell (1821-1910), que ingresó en el Geneva Medical College en 1847, a pesar del desacuerdo de la mayoría del profesorado. Éste se tradujo, al cabo de unos años, en la prohibición de la matriculación de más mujeres en dicho college. Por otra parte, la obra The Ways of Women in Their Physical, Moral and Intellectual Relations, by a Medical Man se hace eco de los impedimentos que sufrieron las mujeres que querían estudiar medicina hacia finales del siglo XIX, llegando a decir literalmente: "A standing army of medical men have opposed the movement. They have thrown every imaginable obstacle in the way. Not only have they refused to admit them as pupils into schools of medicine, but they have denounced and ridiculed those who have expressed sympathy for them in their desire to be medically educated" (1873: 404).
} 
desempeñar su práctica profesional, ya que su entorno, desacostumbrado como estaba a ser tratado por doctoras, mostraba desconfianza hacia ellas y prefería ser atendido por sus homólogos del sexo opuesto. Las siguientes palabras del protagonista masculino dejan ver lo poco familiarizado que estaba con este hecho: "I never knew a lady physician before" (Phelps, 1987: 73). En estas condiciones, no es de extrañar que Zay decidiera vivir su vida soltera, lo que metafóricamente sería "consagrarse a la diosa Artemisa", ya que su trabajo le absorbía el tiempo que los cuidados a un esposo y familia le requerían a las mujeres "convencionales".

Dada la diligencia que la actividad diaria de la doctora le exigía, ya desde el principio se caracteriza, como Atalanta, por su agilidad y presteza: "She came suddenly out and down the grass-grown walk, with the nervous step natural to a person in habitual haste" (Phelps, 1987: 17). Pero como Phelps se proponía que la sociedad de su época aceptara al modelo femenino que ella presenta, aclara que no por desempeñar una profesión su protagonista perdía encanto femenino, como tampoco le ocurrió a Atalanta por ser tan atlética: "She had the decisive step which only women of business acquire to whom each movement represent dollars, responsibilities, or projects. Yet he [Yorke] liked to see that she had not lost the grace of movement due to her eminently womanly form. She had preserved the curves of femineity" (sic; Phelps, 1987: 97). También compartían ambas una extraordinaria fortaleza, demostrando así que no se trata de un atributo exclusivamente masculino, como se intentaba dar a entender entonces. Así se hace evidente en diversas ocasiones a lo largo de la novela, pero destacamos estas palabras de su casera por resultar ser una de las más hiperbólicas: "It's been an awfully sickly winter, and they've worked her enough to kill five men and ten ministers" (Phelps, 1987: 221).

Para llevar a cabo las tareas derivadas de su profesión, esta singular doctora, que tenía que desplazarse a caballo por insólitos parajes, no podía vestirse como requería el ideal victoriano. Phelps también explicaba directamente en otros escritos suyos que los nuevos ámbitos en que las mujeres se estaban desenvolviendo hacían que su indumentaria requiriese ciertas modificaciones. Entre ellos, señalamos esta cita de su libro What to Wear?, donde vuelve a elegir a Atalanta como modelo mitológico para la nueva mujer que se estaba forjando ya a finales del siglo XIX: "The statuesque age... has long gone by. This is the age of the athletes. Atalanta has usurped Penelope's place. Hermione dares at length to leave her long seclusion" (Phelps, 1873: 63).

Al comienzo de Doctor Zay, aparecen los protagonistas en lo que se podría asemejar a la carrera en la que participaron Atalanta y Melanion: el visitante Yorke trata de seguir a caballo a la doctora que le sirve de guía accidental con el fin de encontrar Sherman, pero le resulta prácticamente imposible alcanzar su ritmo. Es entonces cuando Phelps nos ofrece una inversión del famoso mito, ya que no es ahora el hombre quien va dejando manzanas (en el caso de la novela, flores de manzano) en su camino, sino ella: "Suddenly, his eye caught the soft, irregular outline of some small object lying in the dust, a rod or so down the direct road. He drove up to it. As he approached it grew pink, as if it blushed. It was an apple-blossom" (25). ${ }^{5}$ A lo largo de la novela seguirán estando presentes las flores de

\footnotetext{
5 Zay habría tomado la flor del árbol que se describe momentos antes: "Yorke had almost passed unnoticed an apple-tree in full blossom" (Phelps, 1987: 22).
} 
manzano en la consulta de la doctora, con lo que se refuerza la presencia del mito (aunque invertido). Por ejemplo, las encontramos habitualmente en su consulta: "A vase of yellow Austrian glass was on the bureau; it held a spray of apple-blossoms" (Phelps, 1987: 49). La voz narradora se encarga de señalar que dichas flores no pasaron inadvertidas a los ojos de Yorke, es decir, que igual que la Atalanta del mito se entretuvo con las manzanas, Yorke se sentía atraído por estas flores y más concretamente por quien las ponía en su camino: "He looked at the... apple-blossoms in the vase" (Phelps, 1987: 54). Phelps va un poco más allá del mito de nuevo cuando incluye en la novela la imagen de las flores de manzano marchitas sobre la mesa de la doctora Zay. A pesar de que han perdido su vitalidad, como también lo estaba haciendo la protagonista, agotada por tanto trabajo, Yorke, lejos de dejar de sentirse atraído por las flores y la doctora, siente un gran deseo de ayudar a recuperarse a la segunda, o quizá la tentación de aprovechar para adelantarla en la carrera, es decir, para conseguirla en matrimonio. ${ }^{6}$

Además de estas referencias indirectas al mito, existen otras más claras. Así lo hace el propio Yorke para describir cómo evoluciona su "conquista"; él mismo menciona el mito de Atalanta, como observamos hacia la mitad del argumento, cuando reconoce lo difícil que le resulta conseguir su amor: "It is like a modern magazine serial that I should be driving with the caryatid. But I have not overtaken Atalanta. There is the Greek tragedy" (Phelps, 1987: 131). En esta cita se aprecia además otra metáfora mítica para aludir a la doctora Zay: Yorke se refiere a ella como una cariátide. Ya desde el principio de la novela, la doctora Zay es comparada con ella: "The figure of the dainty driver in the phaeton, erect, slender, and blue, sat motionless as a caryatid out of employment" (Phelps, 1987: 22). Esta denominación encierra un doble significado: por una parte representa una estatua de mujer con traje talar que algunos arquitectos de Grecia introdujeron en lugar de una columna; y por otra, el sobrenombre de la diosa Diana. El primer significado ensalza una de las características ya mencionadas de la protagonista: su fortaleza y resistencia para aguantar el peso de la responsabilidad que su profesión conlleva. Donde mejor puede vislumbrarse esta connotación es en la siguiente cita, que tiene lugar después de que la doctora volviera de su trabajo: "Yorke thought of the caryatid lifting marble arms to hold the Temple 'high above our heads"' (Phelps, 1987: 149). El segundo significado de "cariátide" la relaciona de nuevo con el mito de Atalanta, puesto que Diana - diosa guerrera romana que se identificaba con la diosa griega Artemis, hija de Zeus y Leto ${ }^{7}$ representaba la antítesis de Afrodita, quien ayudó a Melanion a conseguir el matrimonio con la primera. Artemis, defensora de la castidad, guarda además muchas semejanzas con el personaje de Zay y con el mensaje que Phelps pretende transmitir por medio de ella. En la obra de Chevalier se le llama "the wild goddess of the woods" y se ofrece su sobrenombre, "Lady of the Wild Beasts" (Chevalier, 1996: 46), lo que clarifica la inmediata identificación que hace Yorke de Zay con una cariátide al verla desenvolverse con tanta soltura y presteza por aquel frondoso bosque. De hecho, a veces, la protagonista es descrita de modo que se le asocia directamente con su vitalidad e incluso con lo salvaje, como ocurre

\footnotetext{
${ }^{6}$ Esta asociación de las flores con la doctora se deja ver claramente en la siguiente cita: "The appleblossoms on the bureau had begun to droop. She herself looked pale" (Phelps, 1987: 63).

${ }^{7}$ Según Jean Chevalier, la Diosa Diana se identificó con la griega Artemis desde el siglo V a.C.
} 
en el extracto siguiente: "She wore something brown, a dress of heavy cloth, and it was trimmed with leopard fur, like that he saw in the sleigh. She did not recover her composure. She was like a beautiful wild creature. Her splendid color and fire mocked him. Who was he that he should think to tame her?" (Phelps, 1987: 227). ${ }^{8}$

Artemis se considera además protectora de las mujeres embarazadas, pues a pesar de ser virgen encarna a la diosa de la natividad. La doctora Zay corresponde a esta otra característica de la diosa en uno de los episodios de la novela en el que ayuda a una joven angustiada por el hecho de haber concebido un hijo de un hombre con quien no está casada. A lo largo de la novela se observa también cómo, al igual que Artemis, la doctora muestra un gran aprecio por los niños, que constituyen uno de sus principales sectores de pacientes junto al de las mujeres. ${ }^{9}$ Son diversas las ocasiones en que la doctora no oculta su debilidad por ellos, como demuestra la gran aflicción que siente cuando muere uno (Phelps, 1987: 113), o la premura con que se dirige a socorrer a otro (Phelps, 1987: 185).

Según Chevalier, Artemis es también diosa de la luna, "wandering like the Moon and sporting in the mountains, while her twin brother, Apollo, became a Sun-god" (Chevalier, 1996: 47). En diversos pasajes de Doctor Zay —así como en otras obras de Phelps- la autora recurre a dicho satélite cuando se quiere poner de relieve la feminidad o se centra la atención en la protagonista (Phelps, 1987: 192).

Volviendo concretamente al mito de Atalanta, llegamos al final de la novela, donde Yorke explica cómo va su relación con la esquiva doctora retomando el paralelismo con la competición que aquél recoge. Dado el agotamiento de la doctora Zay, el protagonista se siente ahora con la misma superioridad que Melanion en una clara referencia al citado mito: "I have overtaken Atalanta this time. She stopped for a leaden apple, ....and I got the start" (Phelps, 1987: 254). Sin embargo, a pesar de la ventaja que ha conseguido, Yorke no quiere hacer uso de ella por los motivos que declara a Zay: "I don't want to feel as if I were taking a sort of —advantage... I shall like to remember, all my life, that you came to me first, of your own accord; that you loved me so much, you would grant me this - little proof" (Phelps, 1987: 258). Al final, la doctora no puede resistirse más a las proposiciones de su amado. No hemos de achacar su decisión automáticamente a la debilidad derivada de su agotamiento físico, sino más bien a haber creído las palabras de Yorke, que le prometían una relación igualitaria en la que su profesión no sólo no representaba un obstáculo para él, sino que aumentaba su admiración y respeto por ella. Sus excepcionales características, como las de Atalanta, hacen que su amor por Zay sea aún más intenso. Así lo expresa en la novela:

I told you long ago that you should not be expected to surrender your profession. I should be ashamed of myself if I could ask it of you. I am proud of you. I feel my heart leap over

\footnotetext{
${ }^{8}$ Además, en diversas ocasiones se le describe vestida con piel de animales salvajes, como por ejemplo en las páginas 223, 227 y 233. En otras, se asocia directamente con dicho tipo de animal, como en este caso: "She seemed to have sprung on him, like a leopardess indeed" (Phelps, 1987: 224).

${ }^{9}$ Phelps es fiel a su propósito de reflejar la realidad tal como es también en este detalle de tener como principales pacientes a mujeres y niños. En el estudio Gender, Work and Medicine. Women and the Medical Division of Labour, se especifica: "Although women were allowed to study medicine by the turn of the [nineteenth] century, in most western countries, their practice was first restricted to children and other women" (Riska y Wegar, 1993: 5).
} 
everything you achieve. It is as if I had done it myself, only that it makes me happier, it makes me prouder. I want you as you are, - - the bravest woman I ever knew, the strongest woman and the sweetest... (Phelps, 1987: 239).

La resolución de la novela, donde se presenta una relación amorosa basada en la igualdad de sus miembros y el respeto y la admiración mutuos, nos recuerda, más que a la versión tradicional del mito de Atalanta, a la interpretación del mismo que han hecho recientemente feministas como Betty Miles, que lo llamaría en 1973 "The New Myth of Atalanta". ${ }^{10}$ Según esta versión, Young John, que representa a Melanion, admirado por la inteligencia de la princesa Atalanta, arde en deseos de conocerla y hablar con ella. ${ }^{11}$ Así, después de preparase concienzudamente para ganarle en la carrera que ofrecía como premio su mano, Young John sólo consigue llegar a la meta al mismo tiempo que Atalanta. No obstante, por haber superado al resto de los contrincantes, el rey le concede el honor de casarse con la princesa, ante lo cual reacciona el joven con una actitud similar a la del Yorke de Phelps, según muestra su respuesta: "Thank you, sir, but I could not possibly marry your daughter unless she wishes to marry me. I have run this race for the chance to talk with Atalanta, and, if she is willing, I am ready to claim my prize" (citado en Fryer, 1978: 204). Atalanta, entonces, sonríe al joven mostrando su satisfacción por haber tenido en cuenta su voluntad, y los dos hablan tanto que, al acabar el día, se habían convertido en buenos amigos. Esta versión del mito, al igual que muchas de las novelas que escritoras como Phelps crearon — anticipándose también en cierto modo al Modernismo-, no tiene un final cerrado, como se aprecia en el siguiente fragmento: "By this time, each of them has had wonderful adventures, and seen marvelous sights. Perhaps some day they will be married, and perhaps they will not. In any case they are friends. And it is certain that they are both living happily ever after" (citado en Fryer, 1978: 205).

Aunque la narración de Doctor Zay tampoco especifica si la relación de los protagonistas acaba en matrimonio o no - ya que termina con el simbólico acercamiento de la doctora hacia Yorke-, parece sugerir que sí, puesto que ya anteriormente Yorke había rechazado un vínculo de simple amistad. No obstante, la novela de Phelps comparte con esta nueva interpretación del mito el gran respeto que los personajes masculinos muestran hacia la opinión y voluntad de las mujeres a las que no sólo aman, sino que además admiran, a pesar - o a causa - de sus cualidades innovadoras con respecto a las de la "True Woman". Yorke, con su actitud, se hace merecedor de compartir la vida con Zay, pues se ajusta a los parámetros que ella exige.

Según el análisis expuesto, podemos llegar a la conclusión de que Phelps pretendió con esta novela —igual que con otras obras suyas - ofrecer a las mujeres de su tiempo un modelo en que fijarse para poder esquivar las restricciones que la sociedad victoriana les imponía, para lo cual recurre a un mito femenino. Su riqueza reside en que no les marca un camino determinado a seguir, sino que las invita a decidir por sí mismas y a no rechazar el amor por decreto, sino a replanteárselo, si éste impide que otras facetas de su vida se desarrollen. El mito de Atalanta le resultó especialmente ilustrativo para su objetivo, y al

\footnotetext{
${ }^{10}$ Miles, Betty. "Atalanta and the Race”, Ms. (Marzo, 1973): 75-78. Citado por Judith Fryer.

${ }^{11}$ Enfatizo el término "inteligencia" para contrastar la novedad que introduce esta nueva versión del mito: es la mente y no el aspecto físico de la mujer lo que atrae al hombre en este caso.
} 
usarlo, no sólo destaca las posibilidades que ofrece la mitología al mundo femenino, sino que se enfrenta al patriarcado en un aspecto más, el de modificar el mito en ciertos aspectos para presentar el mensaje que pretende difundir con él. Así, se adelanta en cierto modo, como hemos visto, a la práctica llevada a cabo por las feministas posteriormente. Merece la pena, pues, que volvamos nuestra mirada hacia obras que, por pertenecer a otras épocas o por aparentar cierta simplicidad, han sido relegadas a un segundo plano o no han sido estudiadas con la profundidad que merecen. Al indagar en dicho análisis, descubrimos que aún queda mucho trabajo por hacer en este sentido, y que los resultados suelen ser reveladores de las circunstancias vitales que rodeaban a quienes escribían estas obras, ayudándonos así a tener una visión más completa de su contexto histórico. De este modo, como nos ha hecho ver el estudio de la obra de Elizabeth Stuart Phelps, Doctor Zay, comprendemos que el mito, la ficción y la realidad están más unidos de lo que podríamos imaginar a simple vista.

\section{REFERENCIAS BIBLIOGRÁFICAS}

BAYM, N., ed. 1999. Norton Anthology of American Literature. New York and London: Norton \& Company.

Bermejo BARRERA, J. C. 1988. El mito griego y sus interpretaciones. Madrid: Ediciones Akal.

Buell, L. 1986. New England Literary Culture: From Revolution to Renaissance. Cambridge, England: Cambridge University Press.

Chevalier, J. and A. Gheerbrant. 1996. Dictionary of Symbols. London, Penguin Books.

Coultrap-McQuin, S. 1990. Doing Literary Business. American Women Writers in the Nineteenth Century. Chapel Hill \& London: The University of Carolina Press.

Downing, C. 1999. La diosa. Imágenes mitológicas de lo femenino. Barcelona: Kairós.

Duin Kelly, L. 1983. The Life and Works of Elizabeth Stuart Phelps, Victorian Feminist Writer. New York: The Whitston Publishing Company.

FrYer, J. 1978. The Faces of Eve. Women in the Nineteenth Century American Novel. Oxford: Oxford University Press.

Gibson, M. 2003. “Atalanta.” Encyclopedia Mythica. 11 dic. 2003. http:// www.pantehon.org/ articles/a/atalanta.html: 1-2.

Gleadle, K. 1995. The Early Feminists. Radical Unitarians and the Emergence of the Women's Rights Movement, 1831-51. New York: Saint Martin's Press.

Kessler, C. F. 1982. Elizabeth Stuart Phelps. Boston: G. K. Hall.

Phelps, E. S. 1980 (1896). Chapters from a Life. Eds. A. BAXter, L. Stein and B. Welter. New York: Arno Press.

1987 (1882). Doctor Zay. New York: The Feminist Press. 
. 1983 (1871). The Silent Partner and "The Tenth of January”. New York: The Feminist Press.

- 1871. "Unhappy Girls". The Independent 27 Jul.: 1.

. 1873. What to Wear? Boston: James R. Osgood.

Riska, E. and K. Wegar. eds. 1993. Gender, Work and Medicine. Women and the Medical Division of Labour. London: SAGE.

Sмiтн, J.V.C. The Ways of Women in Their Physical, Moral and Intellectual Relations, by a Medical Man. 1873. New York: J. P. Jewett \& Co. Publishers.

Stuart Mill, J. 1997 (1869). The Subjection of Women. New York: Dover Publications.

Tompkins, J. 1985. Sensational Designs. The Cultural Work of American Fiction 17901860. New York and Oxford: Oxford University Press.

Welter, B. 1985. Dimity Convictions, The American Woman in the Nineteenth Century. Ohio: Ohio University Press. 